\title{
Computers and arrhythmias: computational approaches to understanding cardiac electrical dynamics
}

\author{
Flavio H. Fenton ${ }^{\mathrm{a}, \mathrm{b}, *}$, Elizabeth M. Cherry ${ }^{\mathrm{b}}$, Harold M. Hastings ${ }^{\mathrm{b}}$, Steven J. Evans ${ }^{\mathrm{a}}$ \\ ${ }^{a}$ The Heart Institute, Beth Israel Medical Center, New York, NY 10003, USA \\ ${ }^{b}$ Department of Physics, Hofstra University, Hempstead, NY 11549, USA
}

\begin{abstract}
Heart disease is one of the most prevalent diseases in the world and is the leading cause of death in industrialized countries. In the United States alone, cardiovascular disease affects about 68 million people, roughly $25 \%$ of the total population, from both sexes, and all races and ages, and costs an estimated $\$ 117$ billion annually. It is currently believed that most dangerous cardiac arrhythmias are due to reentrant wave(s) of electrical activity. In particular, a single scroll wave of action potential can be associated with tachycardia, a rapid heart rhythm that often precedes fibrillation, which is associated with multiple scroll waves and is in general a lethal arrhythmia if it occurs in the ventricles. We present a computational approach used to understand the initiation and evolution of cardiac arrhythmias, which we believe, forms a necessary link between experiment and theory.
\end{abstract}

Keywords: Complex dynamics; Cardiac arrhythmias; Fibrillation; Spiral waves; Nonlinear dynamics; Electrophysiology; Chaos

\section{Introduction}

Cardiovascular disease is the most common cause of death in the industrialized world, with serious health and economic impacts. Nearly one million deaths annually over $40 \%$ of all deaths - are caused by cardiovascular disease in the United States alone. Of these, 250,000 are due to cardiac arrest, with most attributed to ventricular fibrillation (VF) [1]. Chronic atrial fibrillation (AF), while not immediately life-threatening, represents the most commonly encountered cardiac arrhythmia in the clinical setting, with an estimated two million cases in the United States alone, and is responsible for an estimated $15 \%$ of all strokes [1]. Ventricular and atrial fibrillation represent two of the most serious types of arrhythmias, forms of cardiovascular disease in which the normal electrical signal responsible for initiating coordinated contraction of the atria and ventricles becomes disrupted.

Despite the seriousness and prevalence of cardiac fibrillation, treatment at this time is, in general, unsatisfactory. Palliative treatments with antiarrhythmic drugs often do not

* Corresponding author. Tel.: +1 (516) 770-1711; Fax: +1 (516) 463-3059; E-mail: fenton@presto.physics.neu.edu prevent fibrillation. Studies indicate that more than half of sudden cardiac deaths arise in individuals with no previous symptoms [1], so that implantable cardioverter defibrillator devices alone cannot eliminate VF. Furthermore, fibrillation often is complicated by the fact that arrhythmias frequently develop in the context of other cardiovascular disease, such as heart failure, which change the heart's anatomical and electrophysiological properties.

It has become widely accepted that the most dangerous cardiac arrhythmias are due to reentrant waves, which correspond to scroll waves of electrical activity in three dimensions. These waves circulate repeatedly through cardiac tissue at a frequency higher than the heart's pacemaker, thereby altering the heart's regular function and resulting in inadequate pumping. It is currently believed that a single scroll wave of electrical activity can be associated with tachycardia, a rapid heart rhythm that is often a precursor to fibrillation in the ventricles, while multiple scroll waves can be associated with fibrillation, a lethal arrhythmia if it occurs in the ventricles (see Ref. 2 and references therein).

Over the past several years, we have developed a set of robust computational models for conducting basic research on the initiation and evolution of arrhythmias as a function of electrophysiological and anatomical properties. In 
this talk we present some results on initiation, evolution, and interactions of scroll waves in extended systems whose cells possess specific dynamical properties and are assembled into complex anatomies corresponding to real human and animal hearts.

\section{Computational challenges}

Simulating the cardiac electrical system is complex because a number of very different components are involved. To represent cellular electrical behavior, large numbers of variables must be used to identify different ionic processes such as voltage-gated channels, ion pumps and exchangers, and storage mechanisms for intracellular calcium. To represent the anatomical complexities, the cells must be formed into realistic geometrical structures, including significant anisotropy and several distinct cell types (endocardial, midmyocardial, and epicardial cells in ventricles) that can greatly influence the arrhythmia dynamics. As a consequence of including both cellular details and overall anatomy, dynamical behavior can occur over a large range of temporal scales from microseconds to seconds and minutes as well as over spatial scales from microns to centimeters.

To illustrate how quickly the differences in scales leads to substantial computational needs, consider that the entire volume of muscle in a human heart consists of on the order of $4 \times 10^{9}$ cells, each of which is roughly $150 \times 15 \times 15$ microns in size. If each cell is divided into ten segments for computational purposes, roughly $4 \times 10^{10}$ segments are required in total. For each segment, approximately 50 currents and other variables must be computed (with the currents produced by many different ion channels, from 500 to 30,000 in each cell, depending on the cell type); thus $2 \times 10^{12}$ variables are needed for the entire heart. Finally, a time step of roughly $0.01 \mathrm{~ms}$, corresponding to the time scale of one of the fastest ionic processes, is necessary, thus $10^{5}$ time steps are required to simulate one second of physical time. Altogether, a complete, fully resolved model intended to study the ventricular electrical system will require roughly $10^{17}$ operations to simulate just one second of real time [3].

Despite this estimate, progress over the last decade suggests that the level of detail described may not be necessary. Significant advances made in a variety of areas strongly argue that useful virtual ventricular and atrial models can now be constructed using many fewer operations while still maintaining an appropriate level of resolution and detail. In certain circumstances, intermediate phenomenological models [4,5] may be used instead of detailed ionic models that track several ionic species and 10-15 different ionic currents. Detailed digital models of cardiac anatomy have been obtained from dissected animal hearts [6,7] and scanned images [8]. Moreover, a good understanding of the roles of some spatial scales and spatio-temporal dynamics [5,9-12] in the behavior of individual cells and cardiac tissue has been developed. These advances have provided strong evidence that the estimate of $10^{17}$ operations [3] may be unnecessarily large to answer many questions of interest concerning the dynamics of cardiac fibrillation. For instance, the spatial and temporal resolutions in many cases can be coarsened substantially, to approximately $10^{7} \mathrm{com}$ putational nodes and approximately $10^{4}$ time steps, and the number of variables can be roughly halved, leaving a total of $10^{12}$ operations required to simulate one second. We have found that problems in cardiac fibrillation of this magnitude can be addressed using currently existing supercomputer technology.

\section{Results}

Using a variety of ionic cell models within our computational models, we have analyzed and compared a number of different mechanisms for spiral wave breakup that in principle can occur in cardiac tissue as a function of electrophysiological and electro-anatomical effects. For example, Fig. 1 shows how sodium conductance affects the spiral wave's tip trajectory [5]. Spiral wave trajectories are important because some patterns can produce breakup under certain circumstances, such as in the presence of rotational anisotropy formed by the tissue's fiber orientation, where scroll waves that follow trajectories with large pivot turns (hypermeander, Fig. 1E) can induce a twist in the

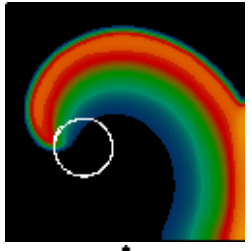

A

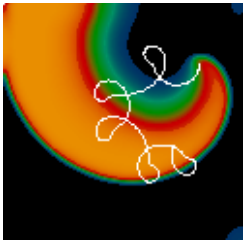

$\mathrm{D}$

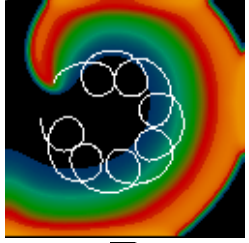

B

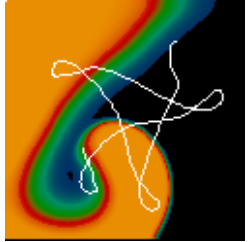

E

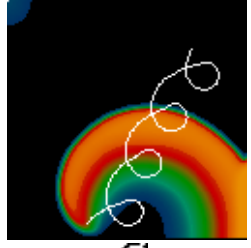

C

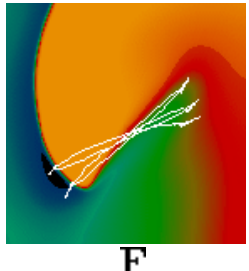

Fig. 1. Varieties of spiral wave tip trajectories. Shown are: (A) circular, (B) epicycloidal, (C) cycloidal, (D) hypocycloidal, (E) hypermeander, and (F) linear trajectory. These trajectories are obtained as the excitability of the tissue is increased, with linear trajectories in the high excitability limit corresponding to normal cardiac tissue. Low excitability occurs in cardiac tissue when it becomes ischemic due to insufficient oxygen. Different spiral trajectories like these have been observed in cardiac tissue experiments [14]. (Figure used with permission from Ref. 5.) 


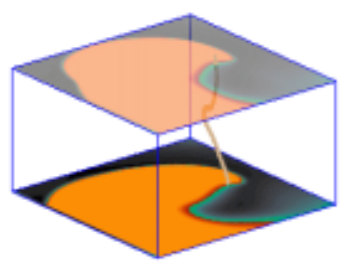

$\Lambda(92 \mathrm{nLs})$

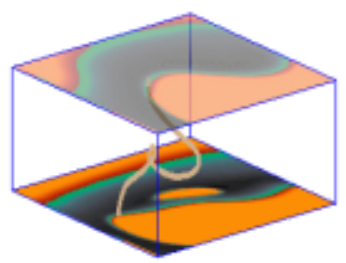

$\mathrm{C}(102 \mathrm{~ns})$

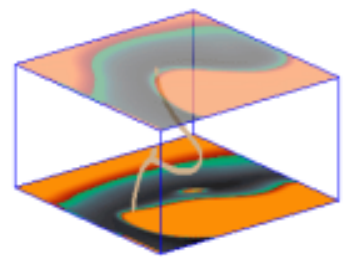

$\mathrm{B}(390 \mathrm{nLs})$

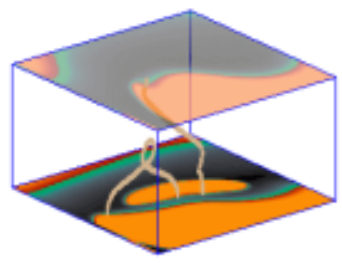

$\mathrm{D}(\perp \perp 11 \mathrm{~s})$

Fig. 2. Breakup and complex dynamics due to filament twist in a simulated three-dimensional slab of cardiac tissue with fiber rotation. An initially straight filament (A) rotates and evolves, accumulating twist and elongating $(\mathrm{B}, \mathrm{C})$ until it touches the boundary and breaks into two (D). Simulated slab has dimensions $4.3 \times 4.3 \times 0.645 \mathrm{~cm}$ (vertical dimension has been stretched by a factor of 2 for visualization). Total fiber rotation is $180^{\circ}$ with anisotropy ratio $5: 1$. (Figure used with permission from Ref. [5].)

nonstationary 3D vortex line around which the scroll wave rotates [4]. When sufficient fiber rotation is present over a given thickness of cardiac tissue, the twist causes the vortex line to elongate, and as the vortex line collides with the tissue boundaries, new scroll waves are generated (see Fig. 2). Along with others, we also have explained how the cycle adaptation of cardiac cell dynamics can lead to a restitution curve that can destabilize scroll waves by alternans and conduction block via a Hopf bifurcation [5].
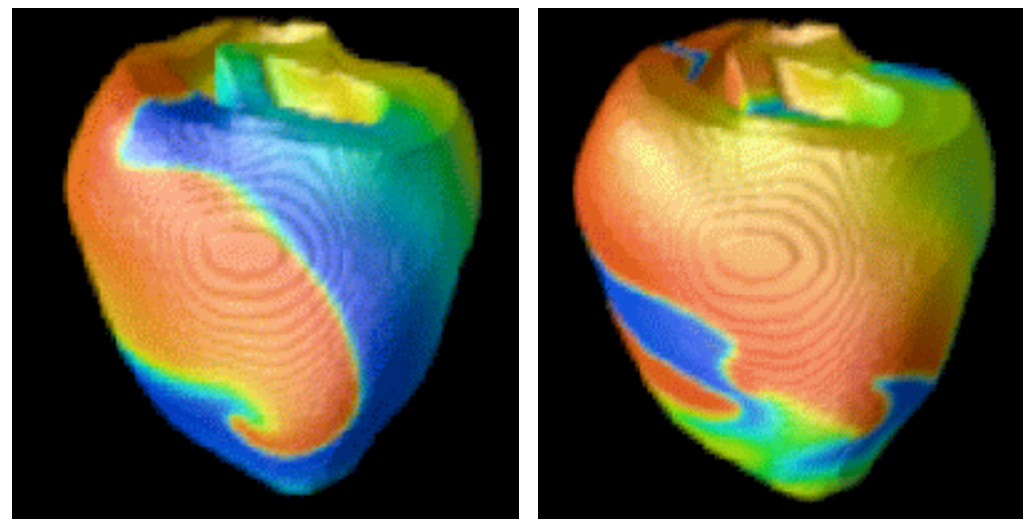

Fig. 3. Simulated tachycardia and fibrillation in rabbit ventricles. (A) Single spiral wave reproducing the electrical activity commonly recorded during tachycardia. (B) Complex dynamics from multiple spiral waves reproducing the dynamics during fibrillation. Blue shows tissue that is electrically polarized (about $-90 \mathrm{mV}$ ) and red tissue that is depolarized (about $15 \mathrm{mV}$ ). 


\section{References}

[1] American Heart Association. 2002 Heart and Stroke Statistical Update. Dallas: American Heart Association, 2001.

[2] Winfree AT. Evolving perspectives during 12 years of electrical turbulence. Chaos 1998;8:1-19.

[3] Wikswo JP, Jr. The Challenges of Spatial Scales in Modeling and Understanding Cardiac Fibrillation. Chicago 2000 World Congress on Medical Physics and Biomedical Engineering, 2000, CD ROM, paper no. 4450-75803.

[4] Fenton F, Karma A. Vortex dynamics in three-dimensional continuous myocardium with fiber rotation: Filament instability and fibrillation. Chaos 1998;8:20-47.

[5] Fenton FH, Cherry EM, Hastings HM, Evans SJ. Multiple mechanisms of spiral wave breakup in a model of cardiac electrical activity. Chaos 2002;12:852-892.

[6] Nielsen PMF, Le Grice IJ, Smaill BH, Hunter PJ. Mathematical model of geometry and fibrous structure of the heart. Am J Physiol 1991;260:H1365-H1378.

[7] Vetter FJ, McCulloch AD. Three-dimensional analysis of regional cardiac function: a model of rabbit ventricular anatomy. Prog Biophys Mol Biol 1998;69:157-183.
[8] Harrild DM, Henriquez CS. A Computer model of normal conduction in the human atria. Circ Res 2000;87:e25-e36.

[9] Courtemanche M, Winfree AT. Re-entrant rotating Waves in a Beeler-Reuter based model of two-dimensional cardiac electrical activity. Int J Bifurcat Chaos 1991;1:431-444.

[10] Karma A. Spiral breakup in model equations of action potential propagation in cardiac tissue. Phys Rev Lett 1993; 71:1103-1106.

[11] Courtemanche M. Complex spiral wave dynamics in a spatially distributed ionic model of cardiac electrical activity. Chaos 1996;6:579-600.

[12] Watanabe M, Fenton F, Evans S, Hastings HM, Karma A. Mechanisms for discordant alternans. J Cardiovasc Electrophysiol 2001;12:196-206.

[13] Fenton F, Cherry EM, Banville I, Gray RA, Hastings HM, Karma A, Evans SJ. Validation of realistic 3D computer models of ventricular arrhythmias with optical mapping experiments (abstract). Pacing and Clinical Electrophysiology 2002; 24 (Part II), 538.

[14] Kim DT, Kwan Y, Lee JJ, Ikeda T, Uchida T, Kamjoo K, Kim YH, Ong JJC, Athill CA, Wu TJ, Czer L, Karagueuzian HS, Chen PS. Patterns of spiral tip motion in cardiac tissue. Chaos 1998;8:137-148. 